\title{
ON THE CONVERGENCE OF EIGENFUNCTIONS TO THRESHOLD ENERGY STATES
}

\author{
THOMAS ØSTERGAARD SØRENSEN AND EDGARDO STOCKMEYER
}

\begin{abstract}
We prove the convergence in certain weighted spaces in momentum space of eigenfunctions of $H=T-\lambda V$ as the energy goes to an energy threshold. We do this for three choices of kinetic energy $T$, namely the nonrelativistic Schrödinger operator, the pseudorelativistc operator $\sqrt{-\Delta+m^{2}}-$ $m$, and the Dirac operator.
\end{abstract}

\section{INTRODUCTION}

In this paper we consider a family of Hamiltonians

$$
H \equiv H(\lambda)=T-\lambda V
$$

where $\lambda>0$ is the coupling constant and $V \geq 0$ is a bounded and integrable potential. We are going to consider different choices of physical kinetic energies $T$ but for the moment, to fix ideas, we set $T=-\Delta$, the Laplace operator in three dimensions. The essential spectrum of $H$ is equal to the interval $[0, \infty)$ and (for $\lambda$ sufficiently large) $H$ has negative discrete eigenvalues $E_{i}<0, i=1,2, \ldots$. We shall henceforth fix an $i \in \mathbb{N}$ and consider the $\lambda$-dependence of $E(\lambda):=E_{i}(\lambda)$. Due to monotonicity, there is a $\lambda_{c} \in \mathbb{R}$ such that, as $\lambda \downarrow \lambda_{c}, E(\lambda) \uparrow 0$. We call $\lambda_{c}$ a coupling constant threshold.

Let $\varphi_{E}=\varphi_{E(\lambda)} \in L_{2}\left(\mathbb{R}^{3}\right)$ be an eigenfunction of $H(\lambda)$ with eigenvalue $E=E(\lambda)$. A detailed study of the behaviour of $E$ as $\lambda \downarrow \lambda_{c}$ for various choices of $T$ was carried out in 13, 14, 16, 12. Here, we are interested in the behaviour of $\varphi_{E}$ as $E \uparrow 0$ (that is, as $\lambda \downarrow \lambda_{c}$ ). It is easy to prove (using closedness of the kinetic energy $T$ ) that if $\varphi_{E}$ converges in $L_{2}\left(\mathbb{R}^{3}\right)$, then the limit function $\varphi_{0}$ is an eigenfunction of $H\left(\lambda_{c}\right)$, i.e., a boundstate with zero energy. If there is no $L_{2}$-convergence, however, we might expect some other kind of convergence of the $\varphi_{E}$ 's. In particular, we are interested in considering the convergence properties of $w(-\mathrm{i} \nabla) \varphi_{E}$ where $w$ is a suitable function of the kinetic energy. (For the question of existence of zero energy eigenstates, see e.g. [1, and the above mentioned papers).

Such questions are, apart from being of independent interest, important for problems pertaining to enhanced binding and the Efimov-effect; see e.g. [4, 21. (Other papers on enhanced binding, using zero-energy 'eigenfunctions' are 2, 3] 8]; these, however, do not use explicitely the convergence properties we discuss here). We shall not comment further on this here. Our work partially use the techniques used in [13, 14, and [1] for the relativistic case (see also 16]). In these papers the authors investigated the relationship between the analytic properties of the eigenvalues near the threshold energy and the existence of eigenvalues at the threshold.

Let us introduce the three different choices of kinetic energy $T$ which we will study in this paper. Let $m>0$ be the mass of the electron.

Date: January 15, 2019.

(C) 2006 by the authors. This article may be reproduced in its entirety for non-commercial purposes. 
Schrödinger case: The free one-particle non-relativistic kinetic energy (in units when $\hbar=1$ ) is given by $-\frac{\Delta}{2 m}$. Choosing units such that $2 m=1$, the operator is just the Laplace-operator in three dimensions mentioned above,

$$
T_{S}:=-\Delta \text {. }
$$

Pseudorelativistiv case: A naïve choice of a free one-particle (pseudo)relativistic kinetic energy is (in units when $\hbar=c=1$ ) given by the pseudodifferential operator,

$$
T_{\psi r e l}:=\sqrt{-\Delta+m^{2}}-m \text {. }
$$

(see e.g. 22] and [9]).

In both of the above cases, assuming that $0 \leq V \in L_{1}\left(\mathbb{R}^{3}\right) \cap L_{\infty}\left(\mathbb{R}^{3}\right)$, the operators $H_{S}(\lambda):=T_{S}-\lambda V$ and $H_{\psi r e l}(\lambda):=T_{\psi r e l}-\lambda V$ are self-adjoint in $L_{2}\left(\mathbb{R}^{3}\right)$ with domains $H^{2}\left(\mathbb{R}^{3}\right)$ and $H^{1}\left(\mathbb{R}^{3}\right)$, respectively, their essential spectrum is $\sigma_{\text {ess }}=$ $[0, \infty)$ and (for large enough $\lambda$ ), they have eigenvalues $E_{i}(\lambda)<0, i \in \mathbb{N}$ (see [17. and [15]).

Dirac case: The free one-particle Dirac operator (again, in units when $\hbar=c=1$ ) is given by

$$
T_{D}:=\alpha \cdot(-i \nabla)+m \beta-m,
$$

acting on $L_{2}\left(\mathbb{R}^{3} ; \mathbb{C}^{4}\right)$. Here $\alpha, \beta$ are the usual Dirac matrices.

If $0 \leq V \in L_{1}\left(\mathbb{R}^{3} ; \mathbb{C}^{4}\right) \cap L_{\infty}\left(\mathbb{R}^{3} ; \mathbb{C}^{4}\right)$ is a (diagonal) potential then $H_{D}(\lambda):=T_{D}-$ $\lambda V$ is self-adjoint with domain $H^{1}\left(\mathbb{R}^{3} ; \mathbb{C}^{4}\right)$, its essential spectrum is $(-\infty,-2 m] \cup$ $[0, \infty)$, and it has eigenvalues $E_{i}(\lambda) \in(-2 m, 0), i \in \mathbb{N}$ (see $[20$ ).

We recall that, for $q \geq 1$, the Banach space $L_{q}\left(\mathbb{R}^{3} ; \mathbb{C}^{4}\right)$ consists of four-component vector functions $\phi=\left(\phi_{1}, \ldots, \phi_{4}\right)^{\mathrm{T}}$ with the norm

$$
\|\phi\|_{L_{q}\left(\mathbb{R}^{3} ; \mathbb{C}^{4}\right)}:=\left(\int_{\mathbb{R}^{3}}\|\phi(\mathbf{x})\|_{\mathbb{C}^{4}}^{q} d \mathbf{x}\right)^{1 / q} .
$$

Here $\|\cdot\|_{\mathbb{C}^{4}}$ is the usual Euclidean norm. Note that since all norms in $\mathbb{C}^{4}$ are equivalent, this norm and

$$
\||| \phi \mid\|_{L_{q}\left(\mathbb{R}^{3} ; \mathbb{C}^{4}\right)}:=\left(\sum_{i=1}^{4}\left\|\phi_{i}\right\|_{L_{q}\left(\mathbb{R}^{3}\right)}^{q}\right)^{1 / q}
$$

are equivalent (for $q=2$ they are equal).

In order to relax the notation we denote by $H(\lambda)=T-\lambda V$ a general Hamiltonian, where $T$ corresponds to one of the three kinetic energies defined above. We will also use the symbol $L_{q}$ for $L_{q}\left(\mathbb{R}^{3}\right)$ or $L_{q}\left(\mathbb{R}^{3} ; \mathbb{C}^{4}\right)$ if there is no risk of confusion; the corresponding norm will be denoted $\|\cdot\|_{q}$. We denote the space of Schwartz-functions (with values in $\mathbb{C}$ or $\mathbb{C}^{4}$ ) by $\mathcal{S}$, and its dual, the space of tempered distributions, by $\mathcal{S}^{\prime}$. The $\left(\mathcal{S}^{\prime}, \mathcal{S}\right)$-pairing is denoted $\langle\cdot, \cdot\rangle$. We define by

$$
\hat{g}(\mathbf{p}):=[\mathcal{F} g](\mathbf{p}):=\frac{1}{(2 \pi)^{3 / 2}} \int_{\mathbb{R}^{3}} e^{-i \mathbf{p} \cdot \mathbf{x}} g(\mathbf{x}) d \mathbf{x}
$$

the Fourier transform of the function $g \in \mathcal{S}\left(\mathbb{R}^{3}\right)$. For four-component vector functions $g=\left(g_{1}, \ldots, g_{4}\right)^{\mathrm{T}}, \hat{g}$ is defined componentwise. For $r \in[1,2]$, the Fourier transform extends to a bounded linear mapping from $L_{r}$ to $L_{r^{\prime}}$, with $1 / r+1 / r^{\prime}=1$. On the other hand, by duality, the Fourier transform extends to $\mathcal{S}^{\prime}$. These two extensions coincide whenever they are both defined.

Consider, for $E \notin \sigma(T)$ and $\left\|\varphi_{E}\right\|_{2}=1$, the eigenvalue equation

$$
(T(-i \nabla)-\lambda V) \varphi_{E}=E \varphi_{E} .
$$

An elementary manipulation shows that this equation can be rewritten as

$$
\varphi_{E}=\lambda(T(-i \nabla)-E)^{-1} V \varphi_{E} .
$$


The latter equation is known (in the Physics literature) as the Lipmann-Schwinger equation.

We recall the following: For $E \notin \sigma(T)$ there is a solution $\varphi_{E}$ of (8) if, and only if, for

$$
\mu_{E}:=V^{1 / 2} \varphi_{E}
$$

the equation

$$
K_{E} \mu_{E}=\lambda^{-1} \mu_{E}
$$

holds, where

$$
K_{E}=V^{1 / 2}(T(-i \nabla)-E)^{-1} V^{1 / 2}
$$

is the Birman-Schwinger operator.

Remark 1. Note that $\lambda_{c} \neq 0$ under the stated assumptions on $V$. For the Schrödinger and pseudorelativistic case, this follows from [19] Theorem (2.3)], for the Dirac case, see [11, Lemma (2.3)].

An interesting feature is that, under fairly general assumptions on the potential $V$, we have the following: If $\lambda_{n} \downarrow \lambda_{c}$ as $n \rightarrow \infty$, and if $\left\{\varphi_{E\left(\lambda_{n}\right)}\right\}_{n \in \mathbb{N}} \subset L_{2}$ is a sequence of corresponding eigenfunctions of $T-\lambda_{n} V$ then there exists a subsequence $\left\{\varphi_{E\left(\lambda_{n_{k}}\right)}\right\}_{k \in \mathbb{N}}$ and a $\mu_{0} \in L_{2}$ such that

$$
\mu_{E\left(\lambda_{n_{k}}\right)} \rightarrow \mu_{0} \quad \text { in } \quad L_{2} \quad \text { as } \quad k \rightarrow \infty,
$$

where $\mu_{E(\lambda)}$ is given by (10).

An analogous result holds for the Dirac operator when $E(\lambda) \downarrow-2 m$ as $\lambda \uparrow \lambda_{c}$ in which case the limiting function is denoted by $\mu_{-2 m}$.

The precise statement of the conditions on $V$ is in Lemma10 in Section 5 where we also give a proof.

Throughout this paper ' $E \rightarrow 0$ ' (' $E \rightarrow-2 m$ ') means to take sequences $\left\{\lambda_{n}\right\}_{n \in \mathbb{N}}$ with $\lambda_{n} \uparrow \lambda_{c}\left(\downarrow \lambda_{c}\right)$ for which $\left\{\mu_{E\left(\lambda_{n}\right)}\right\}_{n \in \mathbb{N}}$ has a limit in $L_{2}$.

From (13) we can construct what will turn out to be the relevant (generalized) zero-energy solution. We call this a 'threshold energy state'.

Let us now state the condition on the weight functions $w$. We denote $x=|\mathbf{x}|$ and $p=|\mathbf{p}|$, and $\chi_{<}:=\chi_{[0,1)}$ and $\chi_{>}:=\chi_{[1, \infty)}$, with $\chi_{A}$ the characteristic function of the set $A$.

Let $w_{S}: \mathbb{R}^{3} \rightarrow \mathbb{C}$ (Schrödinger), $w_{\psi r e l}: \mathbb{R}^{3} \rightarrow \mathbb{C}$ (pseudorelativistic), and $w_{D}: \mathbb{R}^{3} \rightarrow M_{4 \times 4}(\mathbb{C})(4 \times 4$ matrices over $\mathbb{C})$ (Dirac) satisfy

$$
\begin{aligned}
& \frac{w_{S}(\mathbf{p}) \chi_{<}(p)}{p^{2}} \in L_{2}\left(\mathbb{R}^{3}\right) \quad \text { and } \quad \frac{w_{S}(\mathbf{p}) \chi_{>}(p)}{p^{2}} \in L_{\infty}\left(\mathbb{R}^{3}\right), \\
& \frac{w_{\psi r e l}(\mathbf{p}) \chi_{<}(p)}{p^{2}} \in L_{2}\left(\mathbb{R}^{3}\right) \quad \text { and } \quad \frac{w_{\psi r e l}(\mathbf{p}) \chi_{>}(p)}{p} \in L_{\infty}\left(\mathbb{R}^{3}\right), \\
& \frac{\left|w_{D}(\mathbf{p})\right| \chi_{<}(p)}{p^{2}} \in L_{2}\left(\mathbb{R}^{3} ; \mathbb{C}^{4}\right) \quad \text { and } \quad \frac{\left|w_{D}(\mathbf{p})\right| \chi_{>}(p)}{p} \in L_{\infty}\left(\mathbb{R}^{3} ; \mathbb{C}^{4}\right),
\end{aligned}
$$

where in the last expression $\left|w_{D}(\mathbf{p})\right|$ denotes any norm of the matrix $w_{D}(\mathbf{p}$ ) (for instance, its largest eigenvalue, in absolute value). We write in general $w(\mathbf{p})$ for one of the three above defined functions. Our main result in this paper is the following:

Theorem 1. Let $H(\lambda)=T-\lambda V$, with $T$ one of the kinetic energy operators mentioned above, and $V \in L_{1} \cap L_{\infty}$. Let $\lambda_{c}$ be a coupling constant threshold, let $\lambda_{n} \downarrow \lambda_{c}$, and $\left\{\varphi_{n}\right\}_{n \in \mathbb{N}} \subset L_{2}$ such that $H\left(\lambda_{n}\right) \varphi_{n}=E\left(\lambda_{n}\right) \varphi_{n}$. Let $\left\{\mu_{n}\right\}_{n \in \mathbb{N}}$ be the corresponding Birman-Schwinger eigenfunctions defined by (10), and assume that $\mu_{n} \rightarrow \mu_{0}$ in $L_{2}$ as $n \rightarrow \infty$. Define

$$
\varphi_{0}(\mathbf{x}):=\lambda_{c} \int_{\mathbb{R}^{3}} T^{-1}(\mathbf{x}, \mathbf{y}) V^{1 / 2}(\mathbf{y}) \mu_{0}(\mathbf{y}) d \mathbf{y},
$$


where $T^{-1}(\mathbf{x}, \mathbf{y}):=\lim _{E \rightarrow 0}(T-E)^{-1}(\mathbf{x}, \mathbf{y})$. Let finally $w$ satisfy the conditions (14)(16) .

Then

$$
w \hat{\varphi}_{n} \rightarrow w \hat{\varphi}_{0} \quad \text { in } L_{2} \quad \text { as } n \rightarrow \infty .
$$

Furthermore, $\varphi_{0}$ satisfies

$$
H \varphi_{0}=0 \text { in } \mathcal{S}^{\prime}
$$

\section{Remark 2.}

(1) An analogous theorem holds for the Dirac case when $E \rightarrow-2 m$. In that case we define

$$
\varphi_{-2 m}(\mathbf{x}):=\lambda_{c} \int_{\mathbb{R}^{3}}(T+2 m)^{-1}(\mathbf{x}, \mathbf{y}) V^{1 / 2}(\mathbf{y}) \mu_{-2 m}(\mathbf{y}) d \mathbf{y} .
$$

This is the limiting object for which (18) holds, and which turns out to solve $H \varphi_{-2 m}=(-2 m) \varphi_{-2 m}$ in $\mathcal{S}^{\prime}$.

(2) Explicit expressions for $(T-E)^{-1}(\mathbf{x}, \mathbf{y})$ and its limits, for the three choices of kinetic energy $T$, are given in Section 2.3.

(3) Note that not all solutions of $H \varphi_{0}=0$ in the distributional sense have the form (17).

(4) In contrast to the Laplacian, the pseudorelativistic kinetic energy behaves as $p^{2}$ for small (momenta) $p$ and as $p$ for large momenta. The conditions in (15) are enough to ensure that (see (58) below)

$$
\left\|w(\mathbf{p}) \chi_{<}(p) /\left(\sqrt{p^{2}+m^{2}}-m\right)\right\|_{2} \text { and }\left\|w(\mathbf{p}) \chi_{>}(p) /\left(\sqrt{p^{2}+m^{2}}-m\right)\right\|_{\infty}
$$

are finite.

(5) Examples of weight functions are $w_{S}(\mathbf{p})=p^{2 s}, w_{\psi r e l}(\mathbf{p})=\left(\sqrt{p^{2}+m^{2}}-\right.$ $m)^{s}$, and $w_{D}(\mathbf{p})=|\alpha \cdot \mathbf{p}+m \beta-m|^{s}$, all for $s \in\left(\frac{1}{2}, 2\right]$. Thus, in general we have that $w(\mathbf{p})=|T(\mathbf{p})|^{s}, s \in\left(\frac{1}{2}, 2\right]$, satisy the conditions (14)-(16).

(6) In the Schrödinger case, convergence of $\nabla \varphi_{E}$ and $\Delta \varphi_{E}$ is known; see e.g. [21. These cases are covered by our results.

Remark 3. It is important to note that our convergence statements are independent of whether there is an eigenvalue at the threshold when $\lambda \rightarrow \lambda_{c}$ or not. Conditions for the limit function $\varphi_{0}\left(\right.$ or $\left.\varphi_{-2 m}\right)$ to be in $L_{2}$ are well known and we list them here for completeness (we thank A. Jensen for commenting this to us).

- Schrödinger 10 13 and pseudorelativistic [16] case: $\varphi_{0} \in L_{2}\left(\mathbb{R}^{3}\right)$ if, and only if, $\int_{\mathbb{R}^{3}} V(\mathbf{x}) \varphi_{0}(\mathbf{x}) d \mathbf{x}=0$.

- Dirac case 12$]: \varphi_{0} \in L_{2}\left(\mathbb{R}^{3}\right)$ if, and only if, $\int_{\mathbb{R}^{3}} V(\mathbf{x}) \beta_{+} \varphi_{0}(\mathbf{x}) d \mathbf{x}=0$. (Or $\int_{\mathbb{R}^{3}} V(\mathbf{x}) \beta_{-} \varphi_{-2 m}(\mathbf{x}) d \mathbf{x}=0$ for $\left.\varphi_{-2 m}\right)$. Here, $\beta_{ \pm}:=(1 \pm \beta) / 2$.

In case $\varphi_{0} \notin L_{2}, \varphi_{0}$ is called a zero resonance, or a half-bound state (see e.g. [10]).

\section{Preliminaries}

2.1. Additional tools for the Dirac operator. We define

$$
T_{D}(\mathbf{p}):=\mathcal{F} T_{D} \mathcal{F}^{-1}=\alpha \cdot \mathbf{p}+m \beta-m .
$$

To study the Dirac case, we introduce the Foldy-Wouthuysen transformation [5, 20 . $U_{\mathrm{FW}}: L_{2}\left(\mathbb{R}^{3} ; \mathbb{C}^{4}\right) \rightarrow L_{2}\left(\mathbb{R}^{3} ; \mathbb{C}^{4}\right)$ which has the property that

$$
U_{\mathrm{FW}} T_{D} U_{\mathrm{FW}}^{-1}=\beta \sqrt{-\Delta+m^{2}}-m .
$$

In momentum space $\hat{U}_{\mathrm{FW}}:=\mathcal{F} U_{\mathrm{FW}} \mathcal{F}^{-1}$ is given by the matrix-valued multiplication operator

$$
\hat{U}_{\mathrm{FW}}(\mathbf{p}):=a_{+}(p)+\beta \alpha \cdot \frac{\mathbf{p}}{p} a_{-}(p),
$$


where

Noting that

$$
a_{ \pm}(p)=\sqrt{\frac{1}{2}\left(1 \pm \frac{m}{\sqrt{p^{2}+m^{2}}}\right)} .
$$

$$
\hat{U}_{\mathrm{FW}}(\mathbf{p})^{-1}=a_{+}(p)-\beta \alpha \cdot \frac{\mathbf{p}}{p} a_{-}(p),
$$

we see that $\hat{U}_{\mathrm{FW}}(\mathbf{p})$ is an orthogonal matrix for every $\mathbf{p} \in \mathbb{R}^{3}$. Therefore, by the definition (5) we have the following:

Lemma 1. For $q \geq 1$, the mapping $\hat{U}_{\mathrm{FW}}: L_{q}\left(\mathbb{R}^{3} ; \mathbb{C}^{4}\right) \rightarrow L_{q}\left(\mathbb{R}^{3} ; \mathbb{C}^{4}\right)$ with $\hat{U}_{\mathrm{FW}}(\mathbf{p})$ given in (23) is an isometry.

Also note that from (21) and (22) follows that

$$
\hat{U}_{\mathrm{FW}}(\mathbf{p}) T_{D}(\mathbf{p}) \hat{U}_{\mathrm{FW}}^{-1}(\mathbf{p})=\mathcal{F} U_{\mathrm{FW}} T_{D} U_{\mathrm{FW}}^{-1} \mathcal{F}^{-1}=\beta \sqrt{p^{2}+m^{2}}-m,
$$

and so, by the spectral theorem (for matrices),

$$
\begin{aligned}
\hat{U}_{\mathrm{FW}}(\mathbf{p})\left(T_{D}(\mathbf{p})-E\right)^{-1} \hat{U}_{\mathrm{FW}}^{-1}(\mathbf{p})=\left(\beta \sqrt{p^{2}+m^{2}}-m-E\right)^{-1} \\
\quad=\left(\begin{array}{cc}
\left(\sqrt{p^{2}+m^{2}}-m-E\right)^{-1} I_{2 \times 2} & 0_{2 \times 2} \\
0_{2 \times 2} & \left(-\sqrt{p^{2}+m^{2}}-m-E\right)^{-1} I_{2 \times 2}
\end{array}\right) \\
=\beta_{+}\left(\sqrt{p^{2}+m^{2}}-m-E\right)^{-1}+\beta_{-}\left(-\sqrt{p^{2}+m^{2}}-m-E\right)^{-1} \\
\equiv \beta_{+} h_{E}^{+}(p)+\beta_{-} h_{E}^{-}(p),
\end{aligned}
$$

where $\beta_{ \pm}:=(1 \pm \beta) / 2$. Equation (27) makes manifest the fact that the problems $E \rightarrow 0$ and $E \rightarrow-2 m$ are symmetric.

In order to perform $L_{q}$-estimates in the Dirac case we need the following lemma, which is a Hölder inequality for matrix-valued functions:

Lemma 2. Let $A: \mathbb{R}^{3} \rightarrow M_{4 \times 4}(\mathbb{C}), g: \mathbb{R}^{3} \rightarrow \mathbb{C}^{4}$. Then, for $1 / q=1 / r+1 / s$,

$$
\|A g\|_{L_{q}\left(\mathbb{R}^{3} ; \mathbb{C}^{4}\right)} \leq\left\|\lambda_{\max }(A)\right\|_{L_{r}\left(\mathbb{R}^{3}\right)}\|g\|_{L_{s}\left(\mathbb{R}^{3} ; \mathbb{C}^{4}\right)}
$$

where $\lambda_{\max }(A)(\mathbf{x}):=\|A(\mathbf{x})\|_{\mathcal{B}\left(\mathbb{C}^{4}\right)}$ is the largest eigenvalue (in absolute value) of the matrix $A(\mathbf{x})$.

Proof. Let $\mathcal{G}(\mathbf{x})=\|A(\mathbf{x}) g(\mathbf{x})\|_{\mathbb{C}^{4}}, \mathcal{A}(\mathbf{x})=\|A(\mathbf{x})\|_{\mathcal{B}\left(\mathbb{C}^{4}\right)}, \mathfrak{g}(\mathbf{x})=\|g(\mathbf{x})\|_{\mathbb{C}^{4}}$, then

$$
\mathcal{G}(\mathrm{x}) \leq \mathcal{A}(\mathbf{x}) \mathfrak{g}(\mathbf{x}) \text { for all } \mathrm{x} \in \mathbb{R}^{3},
$$

and so this, (5), and Hölder's inequality implies that

$$
\begin{aligned}
\|A g\|_{L_{q}\left(\mathbb{R}^{3} ; \mathbb{C}^{4}\right)} & =\left(\int_{\mathbb{R}^{3}}\|A(\mathbf{x}) g(\mathbf{x})\|_{\mathbb{C}^{4}}^{q} d x\right)^{1 / q}=\|\mathcal{G}\|_{L_{q}\left(\mathbb{R}^{3}\right)} \\
& \leq\|\mathcal{A} \mathfrak{g}\|_{L_{q}\left(\mathbb{R}^{3}\right)} \leq\|\mathcal{A}\|_{L_{r}\left(\mathbb{R}^{3}\right)}\|\mathfrak{g}\|_{L_{s}\left(\mathbb{R}^{3}\right)} \\
& =\left\|\lambda_{\max }(A)\right\|_{L_{r}\left(\mathbb{R}^{3}\right)}\|g\|_{L_{s}\left(\mathbb{R}^{3} ; \mathbb{C}^{4}\right)} .
\end{aligned}
$$

2.2. Preliminaries of the proof. For $E \notin \sigma(T)$ we define $f_{E}:=V^{1 / 2} \mu_{E}$ (see also (10)) and if (13) holds we set $f_{0}:=V^{1 / 2} \mu_{0}$ and $f_{-2 m}:=V^{1 / 2} \mu_{-2 m}$, respectively. We rewrite the Lipmann-Schwinger equation (9) as

$$
\varphi_{E}=\lambda(T(-i \nabla)-E)^{-1} f_{E} .
$$

The following properties of $f_{E}$ and its Fourier transform $\hat{f}_{E}$ will be important:

Lemma 3. If $V \in L_{1} \cap L_{\infty}$ then $f_{E} \in L_{1} \cap L_{2}$. Moreover, $f_{E} \rightarrow f_{0}$ in $L_{q}$ for any $q \in[1,2]$. Consequently, also $\hat{f}_{E} \rightarrow \hat{f}_{0}$ in $L_{r}$ for any $r \in[2, \infty]$.

Remark 4. An analogous result holds when $E \rightarrow-2 m$, with $f_{0}$ replaced by $f_{-2 m}$. 
Proof. By Lemma 10] below we have that $\mu_{E} \rightarrow \mu_{0}$ in $L_{2}$ as $E \rightarrow 0$ for our choice of the potential $V$. Using that $V^{1 / 2} \in L_{2} \cap L_{\infty}$ we have, for $E \leq 0$, that $f_{E} \in L_{1} \cap L_{2}$ since

$$
\left\|f_{E}\right\|_{1} \leq\left\|V^{1 / 2}\right\|_{2}\left\|\mu_{E}\right\|_{2}, \quad\left\|f_{E}\right\|_{2} \leq\left\|V^{1 / 2}\right\|_{\infty}\left\|\mu_{E}\right\|_{2} .
$$

In particular we have, for $r \in[1,2]$ and $q=2 r /(2-r)$, that

$$
\left\|f_{E}-f_{0}\right\|_{r} \leq\left\|V^{1 / 2}\right\|_{q}\left\|\mu_{E}-\mu_{0}\right\|_{2} \rightarrow 0 \quad \text { as } \quad E \rightarrow 0,
$$

i.e., $\left\|f_{E}-f_{0}\right\|_{r} \rightarrow 0$ for any $r \in[1,2]$. Finally using the Hausdorff-Young inequality (see e.g. 15] Theorem 5.7]) we get the desired result. In the Dirac case the Hölder inequalities used in (32) and (33) should be understood in the sense explained in Lemma 2

2.3. The kernels of $(T-E)^{-1}$ and the eigenfunctions in coordinate space. In order to have explicit expressions for (31) in coordinates we need to recall the kernels in x-space of the operators $(T-E)^{-1}$ for $E \notin \sigma(T)$.

For the Schrödinger case we have the well-known expression (see e.g. [17])

$$
\left(T_{S}-E\right)^{-1}(\mathbf{x}, \mathbf{y})=\frac{1}{4 \pi} \frac{e^{-\sqrt{|E|}|\mathbf{x}-\mathbf{y}|}}{|\mathbf{x}-\mathbf{y}|} .
$$

For the pseudorelativistic case the kernel can be found in [16]; for completeness we also derive it in Section 4.1. For $\nu_{E}=\sqrt{\left|m^{2}-(E+m)^{2}\right|}$, we have

$$
\begin{aligned}
\left(T_{\psi r e l}-E\right)^{-1}(\mathbf{x}, \mathbf{y}) & =\frac{(E+m) e^{-\nu_{E}|\mathbf{x}-\mathbf{y}|}}{4 \pi|\mathbf{x}-\mathbf{y}|}+\frac{m}{2 \pi^{2}} \frac{K_{1}(m|\mathbf{x}-\mathbf{y}|)}{|\mathbf{x}-\mathbf{y}|} \\
& +\left(m^{2}-\nu_{E}^{2}\right)\left[\frac{m}{2 \pi^{2}} \frac{K_{1}(m|\cdot|)}{|\cdot|} * \frac{e^{-\nu_{E}|\cdot|}}{4 \pi|\cdot|}\right](\mathbf{x}-\mathbf{y}),
\end{aligned}
$$

where $K_{1}$ is a modified Bessel function of the third kind.

In the Dirac case the kernel is computed in [20]; it is given by

$$
\left(T_{D}-E\right)^{-1}(\mathbf{x}, \mathbf{y})=\frac{e^{-\sqrt{\nu_{E}}}}{4 \pi}\left(\frac{m \beta+m+E}{|\mathbf{x}-\mathbf{y}|}+\frac{i \nu_{E} \alpha \cdot(\mathbf{x}-\mathbf{y})}{|\mathbf{x}-\mathbf{y}|}+\frac{i \alpha \cdot(\mathbf{x}-\mathbf{y})}{|\mathbf{x}-\mathbf{y}|^{3}}\right)
$$

with $\nu_{E}$ as before $\left(\nu_{E}=\sqrt{m^{2}-(E+m)^{2}}\right.$ since $\left.E \in(-2 m, 0)\right)$.

Thus for $E \notin \sigma(T)$, in coordinate space we write in general (see (31))

$$
\varphi_{E}(\mathbf{x})=\lambda \int_{\mathbb{R}^{3}}(T-E)^{-1}(\mathbf{x}, \mathbf{y}) f_{E}(\mathbf{y}) d \mathbf{y}
$$

where as usual $T$ is one of our choices of kinetic energy.

In order to make the connection to the threshold energy states we have the following lemma:

Lemma 4. For $E \notin \sigma(T)$ let $\varphi_{E}$ be given pointwise by (37) with one of the choices of kernels of $(T-E)^{-1}$ given in (34)-(36), and let $\varphi_{0}$ be given by (17).

Then, as $E \rightarrow 0$, we have that $\varphi_{E} \rightarrow \varphi_{0}$ in $\mathcal{S}^{\prime}$. Moreover, $V \varphi_{E} \rightarrow V \varphi_{0}$ in $\mathcal{S}^{\prime}$. Case by case $\varphi_{0}$ is given explicitly by:

Schrödinger case:

$$
\varphi_{0}(\mathbf{x})=\frac{\lambda_{c}}{4 \pi} \int_{\mathbb{R}^{3}} \frac{1}{|\mathbf{x}-\mathbf{y}|} f_{0}(\mathbf{y}) d \mathbf{y} .
$$

Pseudorelativistic case:

$$
\begin{aligned}
\varphi_{0}(\mathbf{x})=\lambda_{c} \int_{\mathbb{R}^{3}}\{ & \frac{m}{4 \pi|\mathbf{x}-\mathbf{y}|}+\frac{m}{2 \pi^{2}} \frac{K_{1}(m|\mathbf{x}-\mathbf{y}|)}{|\mathbf{x}-\mathbf{y}|} \\
& \left.+m^{2}\left[\frac{m}{2 \pi^{2}} \frac{K_{1}(m|\cdot|)}{|\cdot|} * \frac{1}{4 \pi|\cdot|}\right](\mathbf{x}-\mathbf{y})\right\} f_{0}(\mathbf{y}) d \mathbf{y} .
\end{aligned}
$$


Dirac case:

$$
\varphi_{0}(\mathbf{x})=\frac{\lambda_{c}}{4 \pi} \int_{\mathbb{R}^{3}}\left(\frac{2 m \beta_{+}}{|\mathbf{x}-\mathbf{y}|}+\frac{i \alpha \cdot(\mathbf{x}-\mathbf{y})}{|\mathbf{x}-\mathbf{y}|^{3}}\right) f_{0}(\mathbf{y}) d \mathbf{y} .
$$

Remark 5. In the case $E \rightarrow-2 m$ the limit function $\varphi_{-2 m}$ is given by

$$
\varphi_{-2 m}(\mathbf{x})=\frac{\lambda_{c}}{4 \pi} \int_{\mathbb{R}^{3}}\left(\frac{-2 m \beta_{-}}{|\mathbf{x}-\mathbf{y}|}+\frac{i \alpha \cdot(\mathbf{x}-\mathbf{y})}{|\mathbf{x}-\mathbf{y}|^{3}}\right) f_{-2 m}(\mathbf{y}) d \mathbf{y}
$$

Proof. By Lemma 7 below the functions $\varphi_{0}$ in (38)-(40) are well defined in $L_{1}+$ $L_{\infty} \subset \mathcal{S}^{\prime}$ since $f_{0} \in L_{1} \cap L_{2}$. The statement on the convergence follows from Lemma 8 below, using Lemma 3 In the pseudorelativistic case, the conditions of Lemma 7 and Lemma 8 are satisfied due to Lemma 9

2.4. The eigenfunctions in momentum space. Since $\varphi_{E} \in L_{2}$ for $E \notin \sigma(T)$, the expressions in momentum space for $\varphi_{E}$ in (31) are straightforward to derive. In general they are given by

$$
\left[\mathcal{F} \varphi_{E}\right](\mathbf{p})=\hat{\varphi}_{E}(\mathbf{p})=\lambda(T(\mathbf{p})-E)^{-1} \hat{f}_{E}(\mathbf{p}),
$$

where $T(\mathbf{p})$ can be either

$$
T_{S}(\mathbf{p})=p^{2}, \quad T_{\psi r e l}(\mathbf{p})=\sqrt{p^{2}+m^{2}}-m, \quad \text { or } T_{D}(\mathbf{p})=\alpha \cdot \mathbf{p}+m \beta-m,
$$

for the Schrödinger, pseudorelativistic, and Dirac case, respectively. In general, the functions $\varphi_{0}$ are not in $L_{2}$.

Lemma 5. For $E \notin \sigma(T)$ let $\hat{\varphi}_{E}$ be given pointwise by (42) with $T(\mathbf{p})$ one of the choices given in (43). Then, as $E \rightarrow 0$, we have that $\hat{\varphi}_{E} \rightarrow \tilde{\varphi}_{0}:=\lambda_{c} T(\mathbf{p})^{-1} \hat{f}_{0}$ in $\mathcal{S}^{\prime}$. Case by case $\tilde{\varphi}_{0}$ is given explicitly by:

Schrödinger case:

$$
\tilde{\varphi}_{0}(\mathbf{p})=\frac{\lambda_{c}}{p^{2}} \hat{f}_{0}(\mathbf{p})
$$

Pseudorelativistic case:

$$
\tilde{\varphi}_{0}(\mathbf{p})=\frac{\lambda_{c}}{\sqrt{p^{2}+m^{2}}-m} \hat{f}_{0}(\mathbf{p}) .
$$

Dirac case:

$$
\tilde{\varphi}_{0}(\mathbf{p})=\lambda_{c}(\alpha \cdot \mathbf{p}+m \beta-m)^{-1} \hat{f}_{0}(\mathbf{p}) .
$$

Remark 6.

(1) In the case $E \rightarrow-2 m$ the limit function $\tilde{\varphi}_{-2 m}$ is given by

$$
\tilde{\varphi}_{-2 m}(\mathbf{p})=\lambda_{c}(\alpha \cdot \mathbf{p}+m \beta+m)^{-1} \hat{f}_{-2 m}(\mathbf{p}) .
$$

(2) The limit function denoted by $\tilde{\varphi}_{0}$ is in fact the Fourier transform of the function $\varphi_{0}$ defined in Lemma 4 . This is proved in the next section (see (510).

The proof of Lemma 5 is given in Section 4

\section{Proof of Theorem 1}

Now we are ready to prove Theorem 1

Let $\phi \in \mathcal{S}$ then (8) implies that

$$
\left\langle T(-i \nabla) \varphi_{E}, \phi\right\rangle-\lambda\left\langle V \varphi_{E}, \phi\right\rangle=E\left\langle\varphi_{E}, \phi\right\rangle .
$$

Here $\langle\cdot, \cdot\rangle$ is the $\left(\mathcal{S}^{\prime}, \mathcal{S}\right)$-pairing. Note first that $T(-i \nabla) \phi \in \mathcal{S}$. Secondly, due to Lemma 4 we have that $\varphi_{E} \rightarrow \varphi_{0}$ and $V \varphi_{E} \rightarrow V \varphi_{0}$ in $\mathcal{S}^{\prime}$ as $E \rightarrow 0$. Therefore, taking the limit in (48), we get

$$
\left\langle T(-i \nabla) \varphi_{0}, \phi\right\rangle-\lambda_{c}\left\langle V \varphi_{0}, \phi\right\rangle=0,
$$


which proves that $\varphi_{0}$ satisfies $H\left(\lambda_{c}\right) \varphi_{0}=0$ in $\mathcal{S}^{\prime}$. This arguments holds for all three choices of $T$.

Consider the fact that

$$
\left\langle\mathcal{F} \varphi_{E}, \phi\right\rangle:=\left\langle\varphi_{E}, \mathcal{F} \phi\right\rangle .
$$

The function $\mathcal{F} \varphi_{E}$ satisfies (42) and by Lemma 15 converges in $\mathcal{S}^{\prime}$ to the function $\tilde{\varphi}_{0}$ defined in (44)-(46). On the other hand, by Lemma 4 the right side of (50) converges to $\left\langle\varphi_{0}, \mathcal{F} \phi\right\rangle$ as $E \rightarrow 0$. Therefore, taking the limit $E \rightarrow 0$ in (50) we get

$$
\hat{\varphi}_{0}=\mathcal{F} \varphi_{0}=\tilde{\varphi}_{0} \quad \text { in } \mathcal{S}^{\prime} .
$$

It remains to proof that for $w$ satisfying the conditions (14)-(16) we have

$$
w \hat{\varphi}_{E} \rightarrow w \hat{\varphi}_{0} \text { in } L_{2} \text { as } E \rightarrow 0 .
$$

This is carried out in detail now. We start by working with the general expressions. The specific cases are left to the end. The main object of interest is the difference $w \hat{\varphi}_{E}-w \hat{\varphi}_{0}$. This we rewrite using (42) and its counterpart for $E=0$ (now $\hat{\varphi}_{0}=\tilde{\varphi}_{0}=T(\mathbf{p})^{-1} \hat{f}_{0}$ from (44)-(46)). We have

$$
\begin{aligned}
& \left\|w\left(\hat{\varphi}_{E}-\hat{\varphi}_{0}\right)\right\|_{2}=\left\|w\left(\lambda(T(\mathbf{p})-E)^{-1} \hat{f}_{E}-\lambda_{c} T(\mathbf{p})^{-1} \hat{f}_{0}\right)\right\|_{2} \\
& \leq\left\|w\left(\lambda(T(\mathbf{p})-E)^{-1}-\lambda_{c} T(\mathbf{p})^{-1}\right) \hat{f}_{0}\right\|_{2}+\lambda\left\|w(T(\mathbf{p})-E)^{-1}\left(\hat{f}_{E}-\hat{f}_{0}\right)\right\|_{2} .
\end{aligned}
$$

Since $\lambda \rightarrow \lambda_{c}$ as $E \rightarrow 0$ it is enough to prove that

$$
\left\|w\left((T(\mathbf{p})-E)^{-1}-T(\mathbf{p})^{-1}\right) \hat{f}_{0}\right\|_{2} \rightarrow 0 \quad \text { as } \quad E \rightarrow 0,
$$

and

$$
\left\|w(T(\mathbf{p})-E)^{-1}\left(\hat{f}_{E}-\hat{f}_{0}\right)\right\|_{2} \rightarrow 0 \quad \text { as } \quad E \rightarrow 0 .
$$

The term in (54) can be estimated by

$$
\begin{aligned}
\left\|w(T(\mathbf{p})-E)^{-1}\left(\hat{f}_{E}-\hat{f}_{0}\right)\right\|_{2} & \leq\left\|w \chi_{<}(T(\mathbf{p})-E)^{-1}\right\|_{2}\left\|\hat{f}_{E}-\hat{f}_{0}\right\|_{\infty} \\
& +\left\|w \chi_{>}(T(\mathbf{p})-E)^{-1}\right\|_{\infty}\left\|\hat{f}_{E}-\hat{f}_{0}\right\|_{2} .
\end{aligned}
$$

Due to Lemma 3 it is enough to show that the first factors in the two terms on the right side of (55) stay finite as $E \rightarrow 0$, then (54) follows.

Now we prove the convergence statement case by case.

Schrödinger case. We have that (for $E \leq 0$ )

$$
\left(T_{S}(\mathbf{p})-E\right)^{-1}=\left(p^{2}-E\right)^{-1} \leq p^{-2},
$$

therefore the first two factors on the right hand side of (55) are finite by the condition (14). This proves (54).

To prove (53) we use Lebesgue's theorem of dominated convergence, with the function $2|w(\mathbf{p})| \hat{f}_{0} \mid / p^{2}$ as a dominant (see (56) $)$; this is in $L_{2}$ since we can again split in large and small $p$ as in (55) and use the condition (14). Hence, we have proved (52) for the Schrödinger case.

Pseudorelativistic case. We here use that for $E<0$

$$
\left(T_{\psi r e l}(\mathbf{p})-E\right)^{-1}=\frac{1}{\sqrt{p^{2}+m^{2}}-m-E} \leq \frac{1}{\sqrt{p^{2}+m^{2}}-m} .
$$

Additionaly, there exist constants $c_{1}$ and $c_{2}$ such that

$$
\frac{1}{\sqrt{p^{2}+m^{2}}-m} \chi<(p) \leq c_{1} \frac{\chi<(p)}{p^{2}} \text { and } \frac{1}{\sqrt{p^{2}+m^{2}}-m} \chi>(p) \leq c_{2} \frac{\chi_{>}(p)}{p} \text {. }
$$

The finiteness of the first two factors on the right hand side of (55) follows from (57) by using the estimates (58) and the condition (15). This proves (54).

As before, to prove (53) we use Lebesgue's theorem of dominated convergence, with $2|w(\mathbf{p})|\left|\hat{f}_{0}\right| /\left(\sqrt{p^{2}+m^{2}}-m\right)$ as dominant. 
Dirac case. We do the proof for the case $E \rightarrow 0$ and comment on the case $E \rightarrow-2 m$ at the end. Here the general strategy is the same as in the two cases considered above, i.e., we use (55) to prove (54), and Lebesgue's theorem with the dominant given by the zero energy expression to prove (53). The Hölder estimate in (55) should be understood in the sense of Lemma 2 In order to work with diagonal matrices we use the Foldy-Wouthuysen transformation $\hat{U}_{\mathrm{FW}}$ defined in (23). Using (27) we have (with $\tilde{w}=\hat{U}_{\mathrm{FW}} w \hat{U}_{\mathrm{FW}}^{-1}$ )

$$
\left\|w \chi_{<}\left(T_{D}(\mathbf{p})-E\right)^{-1}\right\|_{2} \leq\left\|\tilde{w} \chi_{<} \beta_{+} h_{E}^{+}(p)\right\|_{2}+\left\|\tilde{w} \chi_{<} \beta_{-} h_{E}^{-}(p)\right\|_{2},
$$

where we used Lemma 1 and the fact that $\chi<$ and $\hat{U}_{\mathrm{FW}}(\mathbf{p})$ commute. Analogously, we get

$$
\left\|w \chi_{>}\left(T_{D}(\mathbf{p})-E\right)^{-1}\right\|_{\infty} \leq\left\|\tilde{w} \chi_{>} \beta_{+} h_{E}^{+}(p)\right\|_{\infty}+\left\|\tilde{w} \chi_{>} \beta_{-} h_{E}^{-}(p)\right\|_{\infty} .
$$

The terms with $h_{E}^{+}(p)$ are completely analogous to the pseudorelativistic case (see (27) and (57)-(58) ), except for the fact that the conditions needed for convergence are

$$
\begin{aligned}
\left\|\tilde{w} \chi_{<} \beta_{+} \frac{1}{p^{2}}\right\|_{2} & =\|\| \tilde{w} \beta_{+}\left\|_{\mathcal{B}\left(\mathbb{C}^{4}\right)} \chi<\frac{1}{p^{2}}\right\|_{2}<\infty, \\
\left\|\tilde{w} \chi>\beta_{+} \frac{1}{p}\right\|_{\infty} & =\|\| \tilde{w} \beta_{+}\left\|_{\mathcal{B}\left(\mathbb{C}^{4}\right)} \chi>\frac{1}{p}\right\|_{\infty}<\infty .
\end{aligned}
$$

The terms with $h_{E}^{-}(p)$ are not critical; in fact, for $0 \geq E \geq-m$ we have

$$
\left|h_{E}^{-}(p)\right|=\frac{1}{\sqrt{p^{2}+m^{2}}+m+E} \leq \frac{1}{\sqrt{p^{2}+m^{2}}},
$$

which imply the estimates

$$
\left|h_{E}^{-}(p)\right| \leq \frac{1}{m} \quad \text { and } \quad\left|h_{E}^{-}(p)\right| \leq \frac{1}{p},
$$

and therefore give us the following conditions for convergence:

$$
\begin{aligned}
& \left\|\tilde{w} \chi_{<} \beta_{-}\right\|_{2}=\|\| \tilde{w} \beta_{-}\left\|_{\mathcal{B}\left(\mathbb{C}^{4}\right)} \chi_{<}\right\|_{2}<\infty, \\
& \left\|\tilde{w} \chi_{>} \beta_{-} \frac{1}{p}\right\|_{\infty}=\|\| \tilde{w} \beta_{-}\left\|_{\mathcal{B}\left(\mathbb{C}^{4}\right)} \chi>\frac{1}{p}\right\|_{\infty}<\infty .
\end{aligned}
$$

Since $\beta_{ \pm}$are projections and $\hat{U}_{\mathrm{FW}}(\mathbf{p})$ is an orthogonal matrix, (61) and (64) are fullfilled by (16).

In the case $E \rightarrow-2 m$ we have the following estimates for $-2 m \leq E \leq-m$ :

$$
\left|h_{E}^{-}(\mathbf{p})\right| \leq \frac{1}{\sqrt{p^{2}+m^{2}}-m} \quad \text { and } \quad h_{E}^{+}(\mathbf{p}) \leq \frac{1}{\sqrt{p^{2}+m^{2}}},
$$

i.e., in this case the terms with $h_{E}^{-}$are the ones analogous to the pseudorelativistic case and the terms with $h_{E}^{+}$are noncritical. The conditions (61) and (64) are the same with the substitution $\beta_{ \pm} \mapsto \beta_{\mp}$.

Remark 7. Note that (61) and (64) are slightly more general than (16), but that (16) covers both $E \rightarrow 0$ and $E \rightarrow-2 m$.

\section{Usefull Lemmas}

In this section we prove some technical lemmas; these are not optimal and can easily be further generalized, but they are enough for our purposes.

The following lemma is a special case of the Hardy-Littlewood-Sobolev inequality in three dimensions [15, Theorem 4.3]. 
Lemma 6. Let $\epsilon \in(0,1 / 2), \gamma \in[1,2], g \in L_{1+\epsilon}\left(\mathbb{R}^{3}\right)$, and $f \in L_{q}\left(\mathbb{R}^{3}\right), q=$ $(2-\gamma / 3-1 /(1+\epsilon))^{-1}$.

Then

$$
\left|\int_{\mathbb{R}^{3}} \int_{\mathbb{R}^{3}} f(\mathbf{x}) \frac{1}{|\mathbf{x}-\mathbf{y}|^{\gamma}} g(\mathbf{y}) d \mathbf{x} d \mathbf{y}\right| \leq C_{\gamma}\|f\|_{L_{q}\left(\mathbb{R}^{3}\right)}\|g\|_{L_{1+\epsilon}\left(\mathbb{R}^{3}\right)} .
$$

Lemma 7. Let $\epsilon \in(0,1 / 2), \gamma \in[1,2]$, and $g \in L_{1}\left(\mathbb{R}^{3}\right) \cap L_{1+\epsilon}\left(\mathbb{R}^{3}\right)$, and define

$$
\left(I^{\gamma} g\right)(\mathbf{x}):=\int_{\mathbb{R}^{3}} \frac{1}{|\mathbf{x}-\mathbf{y}|^{\gamma}} g(\mathbf{y}) d \mathbf{y} .
$$

Then $I^{\gamma} g \in L_{1}^{\text {loc }}\left(\mathbb{R}^{3}\right)$, and $\left\|I^{\gamma} g\right\|_{L_{1}(K)} \leq C(\gamma, K)\|g\|_{L_{1+\epsilon}\left(\mathbb{R}^{3}\right)}$ for any compact $K \subset \mathbb{R}^{3}$. Furthermore $I^{\gamma} g=I_{1}^{\gamma} g+I_{2}^{\gamma} g$ with $I_{1}^{\gamma} g \in L_{1}\left(\mathbb{R}^{3}\right), I_{2}^{\gamma} g \in L_{\infty}\left(\mathbb{R}^{3}\right)$.

Proof. Multiply (67) by the characteristic function $\chi_{K}$ and integrate in $\mathbf{x}$. The first statement, and the estimate, follow from Fubini's theorem and Lemma 6

Secondly, for $R>0$, split the integral:

$$
\begin{aligned}
\left(I^{\gamma} g\right)(\mathbf{x}) & =\int_{B_{R}(\mathbf{x})} \frac{1}{|\mathbf{x}-\mathbf{y}|^{\gamma}} g(\mathbf{y}) d \mathbf{y}+\int_{\mathbb{R}^{3} \backslash B_{R}(\mathbf{x})} \frac{1}{|\mathbf{x}-\mathbf{y}|^{\gamma}} g(\mathbf{y}) d \mathbf{y} \\
& =\left(I_{1}^{\gamma} g\right)(\mathbf{x})+\left(I_{2}^{\gamma} g\right)(\mathbf{x}) .
\end{aligned}
$$

For the first term in (68), use [6. Lemma 7.12], which says that for $q \in[1, \infty]$ and $0 \leq \frac{1}{p}-\frac{1}{q}<1-\gamma / 3, I_{1}^{\gamma}$ maps $L_{p}\left(\mathbb{R}^{3}\right)$ continuously into $L_{q}\left(\mathbb{R}^{3}\right)$ with

$$
\left\|I_{1}^{\gamma} g\right\|_{q} \leq C_{\gamma, p, q}\|g\|_{p} .
$$

Use this with $p=q=1$. Then $I_{1}^{\gamma} g \in L_{1}\left(\mathbb{R}^{3}\right)$.

For the second term in (68),

$$
\left|\left(I_{2}^{\gamma} g\right)(\mathbf{x})\right| \leq \int_{\mathbb{R}^{3} \backslash B_{R}(\mathbf{x})} \frac{1}{R^{\gamma}}|g(\mathbf{y})| d \mathbf{y} \leq \frac{1}{R^{\gamma}}\|g\|_{1},
$$

so $I_{2}^{\gamma} g \in L_{\infty}\left(\mathbb{R}^{3}\right)$.

Lemma 8. Let $\epsilon \in(0,1 / 2), \gamma \in[1,2]$, and let $G_{n}, G: \mathbb{R}^{3} \rightarrow \mathbb{R}^{3}, n \in \mathbb{N}$, satisfy $G_{n}(\mathbf{x}) \rightarrow G(\mathbf{x})$ as $n \rightarrow \infty$. Assume there exist $c_{1}, c_{2} \in \mathbb{R}_{+}$such that

$$
\left|G_{n}(\mathbf{x})\right| \leq \frac{c_{1}}{|\mathbf{x}|^{\gamma}} \quad \text { and } \quad|G(\mathbf{x})| \leq \frac{c_{2}}{|\mathbf{x}|^{\gamma}} .
$$

Let $\left\{g_{n}\right\}_{n \in \mathbb{N}} \subset L_{1+\epsilon}\left(\mathbb{R}^{3}\right)$ satisfy $g_{n} \rightarrow g$ in $L_{1+\epsilon}\left(\mathbb{R}^{3}\right)$ as $n \rightarrow \infty$. Define the functions

$$
\left(T_{n}^{\gamma} g_{n}\right)(\mathbf{x}):=\int_{\mathbb{R}^{3}} G_{n}(\mathbf{x}-\mathbf{y}) g_{n}(\mathbf{y}) d \mathbf{y}
$$

and

$$
\left(T^{\gamma} g\right)(\mathbf{x}):=\int_{\mathbb{R}^{3}} G(\mathbf{x}-\mathbf{y}) g(\mathbf{y}) d \mathbf{y} .
$$

Then $V T_{n}^{\gamma} g_{n} \rightarrow V T^{\gamma} g$ in $\mathcal{S}^{\prime}\left(\mathbb{R}^{3}\right)$ for all $V \in L_{\infty}\left(\mathbb{R}^{3}\right)$. In particular, $T_{n}^{\gamma} g_{n} \rightarrow$ $T^{\gamma}$ g in $\mathcal{S}^{\prime}\left(\mathbb{R}^{3}\right)$.

Proof. It follows from Lemma[7] and (69) that $V T_{n}^{\gamma} g_{n}, V T^{\gamma} g \in L_{1}\left(\mathbb{R}^{3}\right)+L_{\infty}\left(\mathbb{R}^{3}\right) \subset$ $\mathcal{S}^{\prime}\left(\mathbb{R}^{3}\right)$. For $\phi \in \mathcal{S}\left(\mathbb{R}^{3}\right) \subset L_{q}\left(\mathbb{R}^{3}\right), q>1$, we have, using Fubini's theorem, that

$$
\begin{aligned}
\left\langle V\left(T_{n}^{\gamma} g_{n}-T^{\gamma} g\right), \phi\right\rangle & =\int_{\mathbb{R}^{6}} \phi(\mathbf{x}) V(\mathbf{x})\left[G_{n}(\mathbf{x}-\mathbf{y})-G(\mathbf{x}-\mathbf{y})\right] g(\mathbf{y}) d \mathbf{x} d \mathbf{y} \\
& +\int_{\mathbb{R}^{6}} \phi(\mathbf{x}) V(\mathbf{x}) G_{n}(\mathbf{x}-\mathbf{y})\left(g_{n}-g\right)(\mathbf{y}) d \mathbf{x} d \mathbf{y} \\
& \equiv I_{1}(n)+I_{2}(n) .
\end{aligned}
$$


We will use Lebesgue's theorem of dominated convergence for $I_{1}(n)$. Lemma 6 shows that the inequality

$$
\left|G_{n}(\mathbf{x}-\mathbf{y})-G(\mathbf{x}-\mathbf{y})\right| \leq \frac{c_{1}+c_{2}}{|\mathbf{x}-\mathbf{y}|^{\gamma}}
$$

provides a dominant, so that $I_{1}(n) \rightarrow 0$ as $n \rightarrow \infty$, since $G_{n}(\mathbf{x}) \rightarrow G(\mathbf{x})$ as $n \rightarrow \infty$.

For $I_{2}(n)$, the first inequality in (69) and Lemma [6 gives that $I_{2}(n) \rightarrow 0$ as $n \rightarrow \infty$, since $g_{n} \rightarrow g$ in $L_{1+\epsilon}\left(\mathbb{R}^{3}\right)$ by assumption.

4.1. The pseudorelativistic kernel. Although [35] is given in [16 we want to sketch its proof. Let us start by noting that (see [15. 7.11 (11)])

$$
\begin{aligned}
\left(\frac{1}{\sqrt{-\Delta+m^{2}}}\right)(\mathbf{x}, \mathbf{y}) & =\frac{m^{2}}{2 \pi^{2}} \int_{0}^{\infty} \frac{t}{t^{2}+|\mathbf{x}-\mathbf{y}|^{2}} K_{2}\left(m\left(t^{2}+|\mathbf{x}-\mathbf{y}|^{2}\right)^{1 / 2}\right) d t \\
& =\frac{m^{2}}{2 \pi^{2}} \int_{m|\mathbf{x}-\mathbf{y}|}^{\infty} \frac{K_{2}(s)}{s} d s=\frac{m}{2 \pi^{2}} \frac{K_{1}(m|\mathbf{x}-\mathbf{y}|)}{|\mathbf{x}-\mathbf{y}|},
\end{aligned}
$$

where in the last step we used that $K_{2}(x) / x=-\left(K_{1}(x) / x\right)^{\prime}$ and that $K_{1}(s) / s \rightarrow 0$ as $s \rightarrow \infty$ (see [7, 8.486.15] and (76) below). On the other hand, we have, with $\nu_{E}=\sqrt{m^{2}-(E+m)^{2}}$ and $E<0$, the operator identity

$$
\begin{aligned}
\frac{1}{\sqrt{-\Delta+m^{2}}-m-E}= & \frac{E+m}{-\Delta+\nu_{E}^{2}}+\frac{1}{\sqrt{-\Delta+m^{2}}} \\
& +\left(m^{2}-\nu_{E}^{2}\right) \frac{1}{\sqrt{-\Delta+m^{2}}} \frac{1}{-\Delta+\nu_{E}^{2}} .
\end{aligned}
$$

The expression in (35) follows by computing the kernel of each summand of (73) separatly, using (34) and(72).

Next we have the following convergence statement for the third summand in (35):

Lemma 9. For $\nu_{E}=\sqrt{m^{2}-(E+m)^{2}}, E<0$, and $\mathbf{x} \in \mathbb{R}^{3}$, we have

$$
\left[\frac{K_{1}(m|\cdot|)}{|\cdot|} * \frac{e^{-\nu_{E}|\cdot|}}{|\cdot|}\right](\mathbf{x}) \rightarrow\left[\frac{K_{1}(m|\cdot|)}{|\cdot|} * \frac{1}{|\cdot|}\right](\mathbf{x}) \quad \text { as } \quad E \rightarrow 0 .
$$

Moreover, there exists a constant $c_{1}>0$ such that

$$
\left[\frac{K_{1}(m|\cdot|)}{|\cdot|} * \frac{e^{-\nu_{E}|\cdot|}}{|\cdot|}\right](\mathbf{x}) \leq\left[\frac{K_{1}(m|\cdot|)}{|\cdot|} * \frac{1}{|\cdot|}\right](\mathbf{x}) \leq \frac{c_{1}}{|\mathbf{x}|} .
$$

Proof. The following properties of the Bessel function $K_{1}$ (see [7 8.446,8.451.6]) are going to be useful: There exist constants $c$ and $\rho$ such that

$$
K_{1}(x) \leq c \frac{e^{-x}}{\sqrt{x}} \text { for } x>\rho,
$$

moreover for $x>0$

$$
K_{1}(x) \leq \frac{1}{x}
$$

Then, by Newton's theorem (see e.g. [15]),

$$
\int_{\mathbb{R}^{3}} \frac{e^{-\nu_{E}|\mathbf{x}-\mathbf{y}|}}{|\mathbf{x}-\mathbf{y}|} \frac{K_{1}(m|\mathbf{y}|)}{|\mathbf{y}|} d \mathbf{y} \leq \int_{\mathbb{R}^{3}} \frac{1}{|\mathbf{x}-\mathbf{y}|} \frac{K_{1}(m|\mathbf{y}|)}{|\mathbf{y}|} d \mathbf{y} \leq \frac{1}{|\mathbf{x}|} \int_{\mathbb{R}^{3}} \frac{K_{1}(m|\mathbf{y}|)}{|\mathbf{y}|} d \mathbf{y} .
$$

The last integral is finite due to (76) and (77); this proves (75). The convergence in (174) follows from Lebesgue's monotone convergence theorem. 
4.2. Proof of Lemma 5. Let $\phi \in \mathcal{S} \subset L_{q}, q \geq 1$, then

$$
\begin{aligned}
\left\langle\left(\mathcal{F} \varphi_{E}-\tilde{\varphi}_{0}\right), \phi\right\rangle & =\int_{\mathbb{R}^{3}}\left[(T(\mathbf{p})-E)^{-1} \hat{f}_{E}(\mathbf{p})-T(\mathbf{p})^{-1} \hat{f}_{0}(\mathbf{p})\right] \cdot \phi(\mathbf{p}) d \mathbf{p} \\
& =\int_{\mathbb{R}^{3}}\left((T(\mathbf{p})-E)^{-1}-T(\mathbf{p})^{-1}\right) \hat{f}_{0}(\mathbf{p}) \cdot \phi(\mathbf{p}) d \mathbf{p} \\
& +\int_{\mathbb{R}^{3}}(T(\mathbf{p})-E)^{-1}\left(\hat{f}_{E}-\hat{f}_{0}\right)(\mathbf{p}) \cdot \phi(\mathbf{p}) d \mathbf{p} \\
& \equiv I_{1}(E)+I_{2}(E) .
\end{aligned}
$$

(In the Dirac case, the '.' is the scalar product in $\mathbb{C}^{4}$ ).

We first consider the Schrödinger and the pseudorelativistic case.

Note that, in both cases, there exist positive constants $c_{<}, c_{>}$such that for all $\mathbf{p} \in \mathbb{R}^{3}$ and $E \leq 0$ (for the pseudorelativistic case, use (58)),

$$
\left|(T(\mathbf{p})-E)^{-1} \phi(\mathbf{p})\right| \leq \frac{c_{<}}{p^{2}} \chi_{<}(\mathbf{p}) \phi(\mathbf{p})+c_{>} \chi_{2}(\mathbf{p}) \phi(\mathbf{p}) .
$$

By Hölder's inequality, this implies that

$$
\left|I_{2}(E)\right| \leq C\left\|\hat{f}_{E}-\hat{f}_{0}\right\|_{\infty}\left(\left\|\chi<\phi / p^{2}\right\|_{1}+\|\phi\|_{1}\right) .
$$

The last factor is finite since $\phi \in \mathcal{S}\left(\mathbb{R}^{3}\right) \subset L_{1}\left(\mathbb{R}^{3}\right)$, and by Lemma 3 the first one goes to zero as $E$ goes to zero, so $I_{2}(E) \rightarrow 0, E \rightarrow 0$.

For $I_{1}$, we use Lebesgue's theorem of dominanted convergence. By arguments similar to the above, the function $c\left(\chi_{<} / p^{2}+\chi_{>}\right) \phi$ is a dominant (for some $c>0$ ) therefore also $I_{1}(E) \rightarrow 0, E \rightarrow 0$.

For the Dirac case,

$$
\left|I_{1}(E)\right| \leq \int_{\mathbb{R}^{3}}\left\|(T(\mathbf{p})-E)^{-1}-T(\mathbf{p})^{-1}\right\|_{\mathcal{B}\left(\mathbb{C}^{4}\right)}\left\|\hat{f}_{0}(\mathbf{p})\right\|_{\mathbb{C}^{4}}\|\phi(\mathbf{p})\|_{\mathbb{C}^{4}} d \mathbf{p} .
$$

Using that $\hat{U}_{\mathrm{FW}}(\mathbf{p})$ is an orthogonal matrix for all $\mathbf{p} \in \mathbb{R}^{3}$, and (27), we have (for $-m \leq E \leq 0)$ that

$$
\begin{aligned}
& \left\|(T(\mathbf{p})-E)^{-1}\right\|_{\mathcal{B}\left(\mathbb{C}^{4}\right)} \\
& \quad=\left\|\left(\begin{array}{cc}
\left(\sqrt{p^{2}+m^{2}}-m-E\right)^{-1} I_{2 \times 2} & 0_{2 \times 2} \\
0_{2 \times 2} & \left(-\sqrt{p^{2}+m^{2}}-m-E\right)^{-1} I_{2 \times 2}
\end{array}\right)\right\|_{\mathcal{B}\left(\mathbb{C}^{4}\right)} \\
& \quad=\left(\sqrt{p^{2}+m^{2}}-m-E\right)^{-1} \leq\left(\sqrt{p^{2}+m^{2}}-m\right)^{-1} .
\end{aligned}
$$

By an argument as above (in the pseudorelativistic case), Lebesgue's theorem on dominated convergence gives that $I_{1}(E) \rightarrow 0, E \rightarrow 0$ also in this case. Also by arguments as above, (79) and the fact that (by Lemma 3) $\hat{f}_{E} \rightarrow \hat{f}_{0}$ in $L_{\infty}$ gives that also $I_{2}(E) \rightarrow 0, E \rightarrow 0$.

Note that a similar argument works for the Dirac case when $E \rightarrow-2 m$; in this case, for $-2 m \leq E \leq-m$,

$$
\begin{aligned}
& \left\|(T(\mathbf{p})-E)^{-1}\right\|_{\mathcal{B}\left(\mathbb{C}^{4}\right)} \\
& \quad=\left\|\left(\begin{array}{cc}
\left(\sqrt{p^{2}+m^{2}}-m-E\right)^{-1} I_{2 \times 2} & 0_{2 \times 2} \\
0_{2 \times 2} & \left(-\sqrt{p^{2}+m^{2}}-m-E\right)^{-1} I_{2 \times 2}
\end{array}\right)\right\|_{\mathcal{B}\left(\mathbb{C}^{4}\right)} \\
& \quad=\left(\sqrt{p^{2}+m^{2}}+m+E\right)^{-1} \leq\left(\sqrt{p^{2}+m^{2}}-m\right)^{-1} .
\end{aligned}
$$




\section{Convergence of Birman-Schwinger operators And EIGENFUnCtions}

We denote the compact operators by $\mathcal{S}_{\infty}$. For $r \geq 1$, we denote by $\mathcal{S}_{r}$ the $r$ 'th Schatten-class of compact operators (which is a norm-closed two-sided ideal in $\mathcal{S}_{\infty}$ ), and $\|\cdot\|_{\mathcal{S}_{r}}$ its norm.

Lemma 10. Let $\epsilon>0$ and assume that $V \geq 0$ satisfies

$$
\begin{aligned}
& \left.V \in L_{3 / 2+\epsilon}\left(\mathbb{R}^{3}\right) \cap L_{3 / 2-\epsilon}\left(\mathbb{R}^{3}\right) \text { and } E<0 \quad \text { (Schrödinger case }\right), \\
& \left.V \in L_{3+\epsilon}\left(\mathbb{R}^{3}\right) \cap L_{3 / 2-\epsilon}\left(\mathbb{R}^{3}\right) \text { and } E<0 \quad \text { (pseudorelativistic case }\right), \\
& V \in L_{3+\epsilon}\left(\mathbb{R}^{3} ; \mathbb{C}^{4}\right) \cap L_{3-\epsilon}\left(\mathbb{R}^{3} ; \mathbb{C}^{4}\right) \text { and } E \in(-2 m, 0) \quad \text { (Dirac case). }
\end{aligned}
$$

Let $\lambda_{c}$ be a coupling constant threshold, and let $\lambda_{n}, E_{n}, \varphi_{E_{n}}$ satisfy $\left(T-\lambda_{n} V\right) \varphi_{E_{n}}=$ $E_{n} \varphi_{E_{n}},\left\|\varphi_{E_{n}}\right\|_{2}=1, \lambda_{n} \downarrow \lambda_{c}$ as $E_{n} \uparrow 0$ (or $\lambda_{n} \uparrow \lambda_{c}$ when $E_{n} \downarrow-2 m$ in the Dirac case). Let finally

$$
K_{E}=V^{1 / 2}(T(-i \nabla)-E)^{-1} V^{1 / 2}
$$

be the the Birman-Schwinger operator, and $\mu_{E_{n}}=V^{1 / 2} \varphi_{E_{n}}$ the Birmin-Schwinger eigenfunctions associated to $\varphi_{E_{n}}$.

Then

(i) $K_{E_{n}}$ is a compact operator.

(ii) The norm-limit $K_{0}:=\lim _{n \rightarrow \infty} K_{E_{n}}$ exists (and, in the Dirac case, $K_{-2 m}:=$ $\lim _{n \rightarrow \infty} K_{E_{n}}$ exists)

(iii) $K_{0}$ (and in the Dirace case, $K_{-2 m}$ ) is compact.

(iv) There exists a subsequence $\left\{\mu_{E_{n_{k}}}\right\}_{k \in \mathbb{N}}$ and $\mu_{0} \in L_{2}$ such that $\mu_{E_{n_{k}}} \rightarrow \mu_{0}$ as $k \rightarrow \infty$ and $K_{0} \mu_{0}=\frac{1}{\lambda_{c}} \mu_{0}$.

Proof. In the Schrödinger and pseudorelativistic cases, it is enough to show that $V^{1 / 2}\left(T(-i \nabla)-E_{n}\right)^{-1 / 2}$ is compact (since $S$ is compact if, and only if, $S^{*} S$ is compact). For this, we will use that operators of the form $f(x) g(-i \nabla)$ belong to $\mathcal{S}_{r}$ if $f, g \in L_{r}, r \in[2, \infty)$, and that furthermore

$$
\|f(x) g(-i \nabla)\|_{\mathcal{S}_{r}} \leq(2 \pi)^{-3 / r}\|f\|_{r}\|g\|_{r},
$$

see [18, Theorem XI.20]. Note that for $E<0$, the function $\left(p^{2}-E\right)^{-1 / 2}$ belongs to $L_{3+\epsilon}\left(\mathbb{R}^{3}\right)$, and $\left(\sqrt{p^{2}+m^{2}}-m-E\right)^{-1 / 2}$ belongs to $L_{6+\epsilon}\left(\mathbb{R}^{3}\right)$. By (85) and the assumptions (81) and (82) on the potential $V$, this implies that the BirmanSchwinger operator $K_{E_{n}}$ is compact in both cases.

To show the statement on convergence, write

$$
\begin{aligned}
S_{E_{n}} & :=V^{1 / 2}\left(T(-i \nabla)-E_{n}\right)^{-1 / 2} \\
& =V^{1 / 2} \mathcal{F}^{-1}\left(T(\mathbf{p})-E_{n}\right)^{-1 / 2} \chi_{<}(p) \mathcal{F}+V^{1 / 2} \mathcal{F}^{-1}\left(T(\mathbf{p})-E_{n}\right)^{-1 / 2} \chi_{>}(p) \mathcal{F} \\
& \equiv S_{n,<}+S_{n,>} .
\end{aligned}
$$

Again using (85), the assumptions (81) and (82) on the potential, and Lebesgue's theorem on dominated convergence, $\left\{S_{n,<}\right\}_{n \in \mathbb{N}}$ is a Cauchy-sequence in the $\mathcal{S}_{r^{-}}$ norm for $r \in[2,3)$ (in both cases), and $\left\{S_{n,>}\right\}_{n \in \mathbb{N}}$ in the $\mathcal{S}_{r}$-norm for $r \in(3, \infty)$ in the Schrödinger case, and for $r \in(6, \infty)$ in the pseudorelativistic case. Therefore both sequences are Cauchy-sequences in the operator norm. Since the set of compact operators is norm-closed, $\lim _{n \rightarrow \infty} S_{n, \gtrless}$ exist, and are compact operators. Therefore $K_{0}:=\lim _{n \rightarrow \infty} K_{E_{n}}$ exists and is compact, in both the Schrödinger and the pseudorelativistc case.

The proof in the Dirac case is essentially the same, only slightly more involved due to the fact that $T_{D}-E$ is not positiv. Note that, using the Foldy-Wouthuysen 
transformation $U_{\mathrm{FW}}$, we have (see (27))

$$
\begin{aligned}
V^{1 / 2}\left(T_{D}-E\right)^{-1} V^{1 / 2} & =V^{1 / 2} U_{F W}^{-1} \mathcal{F}^{-1}\left(\beta \sqrt{p^{2}+m^{2}}-m-E\right)^{-1} \mathcal{F} U_{F W} V^{1 / 2} \\
& =S_{+}^{*} S_{+}-S_{-}^{*} S_{-},
\end{aligned}
$$

with

$$
\begin{aligned}
& S_{+}=\left(\begin{array}{cc}
\left(\sqrt{p^{2}+m^{2}}-m-E\right)^{-1 / 2} I_{2 \times 2} & 0_{2 \times 2} \\
0_{2 \times 2} & 0_{2 \times 2}
\end{array}\right) \mathcal{F} U_{F W} V^{1 / 2}, \\
& S_{-}=\left(\begin{array}{cc}
0_{2 \times 2} & 0_{2 \times 2} \\
0_{2 \times 2} & \left(\sqrt{p^{2}+m^{2}}+m+E\right)^{-1 / 2} I_{2 \times 2}
\end{array}\right) \mathcal{F} U_{F W} V^{1 / 2} ;
\end{aligned}
$$

As before, it suffices to prove that $S_{+}$and $S_{-}$are compact. Note that $U_{F W}$ is bounded, and that both of the functions $\left(\sqrt{p^{2}+m^{2}}-m-E\right)^{-1 / 2}$ and $\left(\sqrt{p^{2}+m^{2}}+\right.$ $m+E)^{-1 / 2}$ belong to $L_{6+\epsilon}$ (since $E \in(-2 m, 0)$ ), and so the same argument as above imply that $S_{+}$and $S_{-}$are compact. It follows that $K_{E_{n}}$ is compact also in the Dirac case. The convergence follows by similar arguments as above.

It remains to prove (iv). Note that $\left\|\mu_{E_{n}}\right\|_{2} \leq C$ since $V \in L_{\infty}$ and $\left\|\varphi_{E_{n}}\right\|_{2}=$ 1. Since $K_{0}$ is compact, there exists a subsequence $\left\{\mu_{E_{n_{k}}}\right\}_{k \in \mathbb{N}}$ such that $\psi:=$ $\lim _{k \rightarrow \infty} K_{0} \mu_{E_{n_{k}}}$ exists. Using (ii) we get that $\left\|K_{E_{n_{k}}} \mu_{E_{n_{k}}}-\psi\right\|_{2} \rightarrow 0$ as $k \rightarrow$ $\infty$. Since $K_{E_{n}} \mu_{E_{n}}=\frac{1}{\lambda_{n}} \mu_{E_{n}}$, and $\lambda_{n} \rightarrow \lambda_{c}$ as $n \rightarrow \infty$, it follows that $\mu_{0}:=$ $\lim _{k \rightarrow \infty} \mu_{E_{n_{k}}}$ exists, and satisfies $K_{0} \mu_{0}=\frac{1}{\lambda_{c}} \mu_{0}$.

Acknowledgement. Both authors thank Semjon Vugalter for suggesting the study of the problem and for discussions. They also thank Arne Jensen for a careful reading of the manuscript. ES thanks Marco Maceda for helpful discussions. Financial support from the EU IHP network Postdoctoral Training Program in Mathematical Analysis of Large Quantum Systems, contract no. HPRN-CT-2002-0027r, is gratefully acknowledged. TØS was partially supported by the embedding grant from The Danish National Research Foundation: Network in Mathematical Physics and Stochastics, and by the European Commission through its 6th Framework Programme Structuring the European Research Area and the contract Nr. RITA-CT2004-505493 for the provision of Transnational Access implemented as Specific Support Action.

\section{REFERENCES}

[1] Rafael D. Benguria and Cecilia Yarur, Sharp condition on the decay of the potential for the absence of a zero-energy ground state of the Schrödinger equation, J. Phys. A 23 (1990), no. 9, 1513-1518.

[2] Isabelle Catto, Pavel Exner, and Christian Hainzl, Enhanced binding revisited for a spinless particle in nonrelativistic QED, J. Math. Phys. 45 (2004), no. 11, 4174-4184.

[3] Isabelle Catto and Christian Hainzl, Self-energy of one electron in non-relativistic QED, J. Funct. Anal. 207 (2004), no. 1, 68-110.

[4] Thomas Chen, Vitali Vougalter, and Semjon A. Vugalter, The increase of binding energy and enhanced binding in nonrelativistic QED, J. Math. Phys. 44 (2003), no. 5, 1961-1970.

[5] Leslie L. Foldy and Siegfried A. Wouthuysen, On the Dirac Theory of Spin 1/2 Particles and its Non-Relativistic Limit, Phys. Rev. Series II, 78 (1950), no. 1, 29-36.

[6] David Gilbarg and Neil S. Trudinger, Elliptic partial differential equations of second order, Classics in Mathematics, Springer-Verlag, Berlin, 2001, Reprint of the 1998 edition.

[7] I. S. Gradshteyn and I. M. Ryzhik, Table of integrals, series, and products, Academic Press [Harcourt Brace Jovanovich Publishers], New York, 1980, Corrected and enlarged edition edited by Alan Jeffrey, Incorporating the fourth edition edited by Yu. V. Geronimus [Yu. V. Geronimus] and M. Yu. Tseytlin [M. Yu. Tseĭtlin], Translated from the Russian.

[8] Christian Hainzl, Vitali Vougalter, and Semjon A. Vugalter, Enhanced binding in nonrelativistic QED, Comm. Math. Phys. 233 (2003), no. 1, 13-26.

[9] Ira W. Herbst, Spectral theory of the operator $\left(p^{2}+m^{2}\right)^{1 / 2}-Z e^{2} / r$, Comm. Math. Phys. 53 (1977), no. 3, 285-294. 
[10] Arne Jensen and Tosio Kato, Spectral properties of Schrödinger operators and time-decay of the wave functions, Duke Math. J. 49 (1979), no. 3, 583-611.

[11] Martin Klaus, Some applications of the Birman-Schwinger principle, Helv. Phys. Acta 55 (1982/83), no. 1, 49-68.

[12] - On coupling constant thresholds and related eigenvalue properties of Dirac operators, J. Reine Angew. Math. 362 (1985), 197-212.

[13] Martin Klaus and Barry Simon, Coupling constant thresholds in nonrelativistic quantum mechanics. I. Short-range two-body case, Ann. Physics 130 (1980), no. 2, 251-281.

[14] _ Coupling constant thresholds in nonrelativistic quantum mechanics. II. Two-cluster thresholds in N-body systems, Comm. Math. Phys. 78 (1980/81), no. 2, 153-168.

[15] Elliott H. Lieb and Michael Loss, Analysis, second ed., Graduate Studies in Mathematics, vol. 14, American Mathematical Society, Providence, RI, 2001.

[16] Marco Maceda, On the Birman-Schwinger principle applied to $\sqrt{-\Delta+m^{2}}-m$, accepted for publication in J. Math. Phys. (2006).

[17] Michael Reed and Barry Simon, Methods of modern mathematical physics. II. Fourier analysis, self-adjointness, Academic Press [Harcourt Brace Jovanovich Publishers], New York, 1975.

[18] _ Methods of modern mathematical physics. III, Academic Press [Harcourt Brace Jovanovich Publishers], New York, 1979, Scattering theory.

[19] Barry Simon, On the absorption of eigenvalues by continuous spectrum in regular perturbation problems, J. Functional Analysis 25 (1977), no. 4, 338-344.

[20] Bernd Thaller, The Dirac equation, Texts and Monographs in Physics, Springer-Verlag, Berlin, 1992.

[21] S. A. Vugal'ter and G. M. Zhislin, The symmetry and Efimov's effect in systems of threequantum particles, Comm. Math. Phys. 87 (1982/83), no. 1, 89-103.

[22] Ricardo A. Weder, Spectral analysis of pseudodifferential operators, J. Functional Analysis 20 (1975), no. 4, 319-337.

(Thomas Østergaard Sørensen) Laboratoire de Mathématiques, Université Paris-Sud BÂt 425, F-91405 Orsay Cedex, France.

(Thomas Østergaard Sørensen, permanent address) Department of Mathematical Sciences, Aalborg University, Fredrik Bajers Vej 7G, DK-9220 Aalborg East, Denmark.

E-mail address: sorensen@math.aau.dk

(Edgardo Stockmeyer) Mathematisches Institut, Universität München, Theresienstrasse 39, D-80333 Munich, Germany.

E-mail address: stock@mathematik.uni-muenchen.de 